\title{
Distributed initialization-free cost-optimal charging control of plug-in electric vehicles for demand management
}

DOI:

10.1109/TII.2017.2685422

\section{Document Version}

Accepted author manuscript

Link to publication record in Manchester Research Explorer

\section{Citation for published version (APA):}

Zhao, T., \& Ding, Z. (2017). Distributed initialization-free cost-optimal charging control of plug-in electric vehicles for demand management. IEEE Transactions on Industrial Informatics, PP(99), [TII-16-1038].

https://doi.org/10.1109/TII.2017.2685422

\section{Published in:}

IEEE Transactions on Industrial Informatics

\section{Citing this paper}

Please note that where the full-text provided on Manchester Research Explorer is the Author Accepted Manuscript or Proof version this may differ from the final Published version. If citing, it is advised that you check and use the publisher's definitive version.

\section{General rights}

Copyright and moral rights for the publications made accessible in the Research Explorer are retained by the authors and/or other copyright owners and it is a condition of accessing publications that users recognise and abide by the legal requirements associated with these rights.

\section{Takedown policy}

If you believe that this document breaches copyright please refer to the University of Manchester's Takedown Procedures [http://man.ac.uk/04Y6Bo] or contact uml.scholarlycommunications@manchester.ac.uk providing relevant details, so we can investigate your claim.

\section{OPEN ACCESS}




\title{
Distributed Initialization-Free Cost-Optimal Charging Control of Plug-In Electric Vehicles for Demand Management
}

\author{
Tianqiao Zhao, Zhengtao Ding, Senior Member, IEEE
}

\begin{abstract}
This paper considers the optimal charging problem of plug-in electric vehicles (PEVs) on demand side management. PEVs provide a promising alternative solution to reduction of environmental pollution and fuel emission. With a large number of PEVs connected to the grid, a well-designed charging coordination approach is needed to release the impacts on the power system. A distributed cooperative control strategy of PEVs is proposed to meet system interests while respecting each PEV's charging constraint. The proposed strategy is distributed, which only needs to be interacted with the neighbouring agents. Our analysis shows that the proposed strategy solves the optimal charging problem of PEVs in an initialization-free approach, which avoids any procedure for initialization during PEVs' charging process. Furthermore, the proposed strategy is robust to the time-varying available charging power and plug-and-play operations. The simulation studies validate the effectiveness of the proposed distributed strategy.
\end{abstract}

Index Terms-Charging control, plug-in electric vehicle, distributed optimization, charging efficiency, consensus algorithm, multiagent system

\section{INTRODUCTION}

$\mathrm{W}$ ITH gradually depleting fossil fuels and deteriorating environmental problems, massive attentions have been focused on energy conservation and emission reduction among the governments and the whole society. It is widely recognized that electric vehicles $(\mathrm{EVs})$ provide a promising alternative to the traditional internal combustion engine (ICE) vehicles, as they can reduce both environmental pollution and greenhouse effect. Enabling this transformation to PEVs will bring potential benefits to the smart grid [1], [2].

The large-scale integration of PEVs may induce both adverse effects and incentives simultaneously on future grids. One of the apparent impacts is that the grid would be destabilized with a higher peak demand due to the PEV integration [3]. On the other hand, the grid can benefit from the integration in load profile levelling and frequency regulation since PEVs can be treated as a flexible load due to their charging property [4]. Additionally, the satisfaction of PEVs' customers should be improved by designing a properly coordination strategy, which can obtain a high acceptance rate of EVs' utilization. The main concerns of PEVs' users are the total charging time, the total charging cost and the state-of-charge (SoC)

T. Zhao and Z. Ding are with the School of Electrical and Electronic Engineering, University of Manchester, Sackville Street Building, Manchester M13 9PL, UK (e-mails: tianqiao.zhao@postgrad.manchester.ac.uk; zhengtao.ding@manchester.ac.uk). at their end of the charging process. Therefore, with the development of vehicle-to-grid (V2G) technology [5], [6], it is important to design efficient energy-management policies to control and optimize the charging process of EVs for the smart grid development [7]. The purpose of this paper is to design a proper cooperative control strategy to maximize benefits and satisfaction of PEVs' customers while satisfying the constraints of PEVs' operations.

The coordination control and demand management problem for PEVs can be formulated as an optimization problem in a centralized manner [8]-[12]. Such kinds of control strategies usually require a control centre, i.e., an aggregator, to receive the charging status of each PEV from the charging station and a powerful computation centre to process numerous information collected from PEVs [13]. However, with a large number of EVs introduced as controllable units, traditional centralized approaches may lose their efficiency due to intractable computation burden, and they are sensitive to singlepoint failures. To overcome those problems, the decentralized PEVs coordination control strategies are presented in [14][16]. In [14], a decentralized approach is proposed for each charging station to regulate its charging power by responding to an external signal. The decentralized approach only collects PEV's own information, which can relieve computation burdens for the control centre. However, a central external signal, i.e., a coordination control signal [17] or a real-time pricing signal [18], is required to send to an aggregator. A fully decentralized strategy only needs local information that is presented in [15], [16]. However, it is difficult to adjust droop coefficients to instantaneous operating conditions real-timely when lacking broaderly available information in practice [19].

To address the above issues, results in literature consider distributed optimal control strategies for EVs' charging control [20]-[22]. In [22], a distributed optimal strategy is proposed for the control of PEVs' charging rate. However, this control strategy relies on a specific initialization procedure during each PEV charging cycle. Furthermore, the existing distributed control strategies are proposed under undirected communication topologies. Compared with directed graphs, the undirected graphs require bi-directional communication networks, which need more communication bandwidth. In this paper, inspired by [23], we focus on designing a novel distributed optimal control strategy to solve PEVs' optimal charging problem. The optimal strategy maximizes the welfare and satisfaction of PEV's customers, i.e., the charging cost and the rate of change of $\mathrm{SoC}$, by minimizing deviations between 


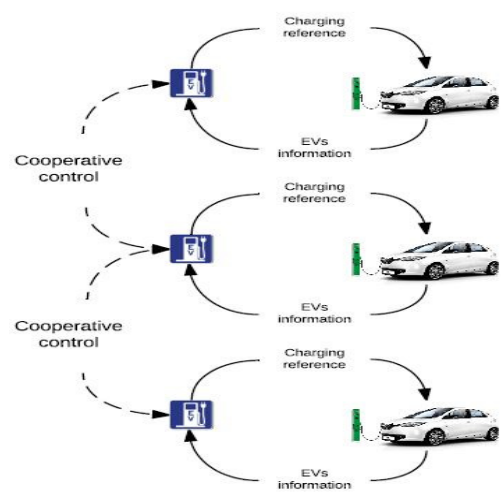

Fig. 1. Distributed demand management for PEVs charging

the charging current and the desired current value under the conditions of individual PEV's charging limit. As shown in Fig. 1, the proposed distributed strategy is implemented based on a multi-agent system (MAS) framework. Each PEV exchanges information with its own neighbours under a directed communication graph. The computational and communication burdens are shared among individual agents (PEVs) based on this distributed scheme, and thus the proposed algorithm can be flexible and scalable. To design a distributed control strategy under a directed graph is much more challenging than under an undirected one, due to the asymmetry of the directed graphs. Our control design consists of two aspects: 1) a consensus algorithm is applied to estimate the mismatches between all PEV allocated powers and the total available charging power on the directed graph; 2) with these mismatch estimations, a nonsmooth analysis based dynamic system is adopted for the dynamical update of the PEV charging power. It should be noted that the developed algorithm can work with time-varying communication topology. Additionally, in the real application, there may exist initialized errors in load satisfaction, the initialization-free is desirable for a practical PEVs charging control. To this end, by characterizing the omega-limit set of the trajectories of our strategy, we show that the proposed algorithm does not require any specific initializing procedure so that PEVs can start from any charging power allocation. Therefore, the proposed distributed optimal control strategy guarantees the plug-and-play operation during the PEV charging process.

The remainder part of the paper is organized as follows. Section II briefly introduces basic preliminaries. Section III provides the problem statement of the coordinated PEV charging control. Section IV formulates the proposed distributed control strategy. Section $\mathrm{V}$ presents the simulation results and corresponding discussions. Finally, the conclusion is drawn in Section VI.

\section{Preliminaries}

In this section, we recall some preliminaries about graph theory, nonsmooth analysis and differential inclusions that are used in this paper. For $l \in \mathbb{R}$, we denote $\mathcal{H}_{l}=\{x \in \mathbb{R} \mid$ $\left.\mathbf{1}_{n}^{T} x=l\right\}$, where $\mathbf{1}_{n}=[1,1 \ldots]^{T} \in \mathbb{R}^{n}$. Let $B(x, \varepsilon)=$ $\left\{y \in \mathbb{R}^{n} \mid\|y-x\|<\varepsilon\right\}$. A set-value map $\mathcal{X}: \mathbb{R}^{n} \rightrightarrows \mathbb{R}^{m}$ associates each value in $\mathbb{R}^{n}$ with a set in $\mathbb{R}^{m}$. For $u \in \mathbb{R},[u]^{+}$ denotes $\max \{0, u\}$. Also, for $B_{0} \in \mathbb{R}^{n}, B_{0}=\left[b_{10}, \ldots, b_{i 0}\right]^{T}$, where $b_{i 0} \neq 0$ denotes $i$ th PEV can receive the information of the total available charging power; $b_{i 0}=0$ otherwise; and $\mathbf{1}_{n}^{T} B_{0}=1$.

\section{A. Graph Theory}

Following [24], a directed graph $\mathcal{G}=(\mathcal{V}, \mathcal{E})$, where $\mathcal{V}=$ $\left\{\nu_{1}, \ldots, \nu_{n}\right\}$ denotes the node set and $\mathcal{E} \in \mathcal{V} \times \mathcal{V}$ is the edge set. If $\left(\nu_{i}, \nu_{j}\right) \in \mathcal{E}$ means node $\nu_{i}$ is a neighbour of node $\nu_{j}$. A directed path is a sequence of nodes connected by edges. A directed graph contains a directed spanning tree if there exists a root node that has directed paths to all other nodes. A directed graph is strongly connected if there exists a directed path that connects any pair of vertices. For a directed graph $\mathcal{G}$, the adjacency matrix $\mathcal{A}=\left[a_{i j}\right]$ in $\mathbb{R}^{n \times n}$ is defined by $a_{i i}=0, a_{i j}=1$ if $\left(\nu_{j}, \nu_{i}\right) \in \mathcal{E}$ and $a_{i j}=0$ otherwise. A weighted graph $\mathcal{G}=(\mathcal{V}, \mathcal{E}, A)$ consists of a digraph $(\mathcal{V}$, $\mathcal{E})$ and an adjacency matrix $\mathcal{A} \in \mathbb{R}_{>0}^{n \times n}$ with $a_{i j}>0$ if and only if $(i, j) \in \mathcal{E}$. The weighted in-degree and out-degree of $i$ are defined as $d_{i n}(i)=\sum_{j=1}^{n} a_{i j}$ and $d_{\text {out }}(i)=\sum_{j=1}^{n} a_{j i}$, respectively. The Laplacian matrix $\mathcal{L}=\left[\mathcal{L}_{i j}\right] \in \mathbb{R}^{n \times n}$ associates with $\mathcal{G}$ is defined as $\mathcal{L}_{i i}=\sum_{j \neq i} a_{i j}$ and $\mathcal{L}_{i j}=-a_{i j}$, $i \neq j . \mathcal{G}$ is defined as weight-balanced if $d_{\text {out }}(\nu)=d_{\text {in }}(\nu)$, for all $\nu \in \mathcal{V}$ iff $\mathbf{1}_{n}^{T} \mathcal{L}=0$ iff $\mathcal{L}+\mathcal{L}^{T}$ is positive semi-definite. If $\mathcal{G}$ is strong connected and weight-balanced, zero is a simple eigenvalue zero of $\mathcal{L}+\mathcal{L}^{T}$. In this case, there is a fact that

$$
x^{T}\left(\mathcal{L}+\mathcal{L}^{T}\right) x \geq \lambda_{2}\left(\mathcal{L}+\mathcal{L}^{T}\right)\left\|x-\frac{1}{n} \mathbf{1}_{n}^{T} \mathbf{1}_{n} x\right\|^{2},
$$

where $\lambda_{2}\left(\mathcal{L}+\mathcal{L}^{T}\right)$ is the smallest non-zero eigenvalue of $\mathcal{L}+$ $\mathcal{L}^{T}$.

\section{B. Nonsmooth Analysis and Differential Inclusions}

Following [25], [26], we present some basic notions of nonsmooth analysis and differential inclusions, respectively. A function $f: \mathbb{R}^{n} \rightarrow \mathbb{R}^{m}$ is locally Lipschitz at $x \in \mathbb{R}^{n}$ if for $y, y^{\prime} \in B(x, \varepsilon),\left\|f(y)-f\left(y^{\prime}\right)\right\| \leq L_{x}\left\|y-y^{\prime}\right\|$, where $L_{x}, \varepsilon \in(0, \infty)$. A function $f: \mathbb{R}^{n} \rightarrow \mathbb{R}^{m}$ is said regular at $x \in \mathbb{R}^{n}$ if, for all $\nu \in \mathbb{R}^{n}$, the right and generalized directional derivatives of $f$ at $x$ in the direction of $\nu$ coincide [26]. A function is regular at $x$, if it is continuously differentiable at $x$. A convex function is regular.

Considering a set-valued map $\mathcal{H}: \mathbb{R}^{n} \rightrightarrows \mathbb{R}^{n}$, a differential inclusion on $\mathbb{R}^{n}$ is defined by

$$
\dot{x} \in \mathcal{H}(x) .
$$

The set of equilibria of (2) is denoted by $\operatorname{Eq}(\mathcal{H})=\{x \in$ $\left.\mathbb{R}^{n} \mid 0 \in \mathcal{H}(x)\right\}$. A locally Lipschitz function $f: \mathbb{R}^{n} \rightrightarrows \mathbb{R}$, the set-valued Lie derivative $L_{\mathcal{H}} f, \mathbb{R}^{n} \rightrightarrows \mathbb{R}$, of $f$ with respect to (2) is defined as

$L_{\mathcal{H}} f=\left\{b \in \mathbb{R} \mid \exists v \in \mathcal{H}(x)\right.$ s.t. $\alpha^{T} v=b$ for all $\left.\alpha \in \partial f(x)\right\}$.

The detailed definitions of nonsmooth analysis and differential inclusions are shown in Appendix. 


\section{Problem Formulation FOR the Battery Charging Problem of PEVs}

We assume that multiple PEVs are plugged into a charging station under a specific optimal charging control to schedule their charging profiles during the total charging duration time $T$. The total charging power capacity is known for the charging station during the PEVs charging period. Each PEV is charged with a constant charging current to reach their desired SoC.

The objective of this paper is to design an optimal control method in terms of the economic factors of the PEV charging process, such as the total charging time, the total charging cost, etc. To address this purpose, the modelling of the PEV battery and its charging property is firstly investigated. The existing results on the PEV battery modelling mainly consider two aspects: a) equivalent circuit models [27], [28] and b) electrochemical models [29]. For the equivalent circuit models, they are mainly used for online estimation and power managements, and the electrochemical models are usually adopted for battery design optimization, health characterization, and health-conscious control.

\section{A. Battery Modelling}

For the purpose of the optimal charging control design, an equivalent circuit model is adopted in [30], which regards a Li-ion battery as an ideal energy storage unit. This model has been validated based on the test data [31]. All the analyses in this paper are based on the approximate battery model that the battery parameters are independent of depth of discharge, SoC and temperature. Such assumptions are widely applied in [22], [32], [33] for the optimization and control design.

The model is described as a constant voltage source in series with a constant resistance to consider the resistive energy losses. This model can be represented as

$$
\begin{gathered}
V_{i}=V_{o, i}+R_{i} I_{i}, \\
\dot{\mu}_{i}=\frac{I_{i}}{Q_{i}},
\end{gathered}
$$

where $V_{i}$ is the terminal voltage, $V_{o, i}$ denotes the open circuit voltage, $R_{i}$ represents the equivalent battery internal resistance, $I_{i}$ is the charging current, $Q_{i}$ represents the battery charge capacity, and $\mu_{i}$ is the battery state of charge SoC, of $i$ th PEV, respectively. Note that $V_{o, i}$ can vary with SoC. In this paper, we treat it as a constant voltage source since the variation of $V_{o, i}$ is very small within $25 \%-90 \%$ SoC for lithium-ion batteries [33].

When a PEV is being charged after plugging in, the power consumed by the $i$ th PEV can be represented by multiplying the terminal voltage and its charging current,

$$
P_{E V, i}=V_{o, i} I_{i}+R_{i} I_{i}^{2},
$$

where $P_{E V, i}$ is the instantaneous charging power. Hence, the battery charging current can be expressed as

$$
I_{i}=\frac{1}{2 R_{i}}\left(\sqrt{4 R_{i} P_{E V, i}+V_{o, i}^{2}}-V_{o, i}\right) .
$$

Note that in our paper, the battery charging current and power are positive during charging process and negative for discharging otherwise.

\section{B. Constraints}

The physical limits are represented by the following constraints.

1) Global Constraint of the Charging Power Allocation: For a charging station, there is a limit that the total amount of charging power of all PEVs should not exceed the total available power $P_{\text {total }}$, which is modeled as a upper bound of the utility's power delivery

$$
\sum_{i=1}^{n} P_{E V, i} \leq P_{\text {total }}
$$

2) Local Constraint of Each PEV: The allocated charging power of each PEV is locally bounded by different physical factors, such as the upper bound of outlet's power output, the charging current's tolerance and the charging level [21]. One local constraint is proposed to map the physical above constraints, i.e.,

$$
0 \leq P_{E V, i} \leq P_{E V, i}^{M}
$$

where $P_{E V, i}^{M}$ is the maximum charging power of $i$ th PEV that considers the above limitations.

\section{Optimization Problem Formulation of PEVs}

The charging time length over the charging duration $T_{i}$ of $i$ th PEV is denoted by $\Delta T$, and the time slots is expressed as $K_{i}=\frac{T_{i}}{\Delta T}, k \in K_{i}:=\left\{1, \ldots, K_{i}\right\}$.

In [34], a real-time price model is formulated as the derivative of the generation supply cost, which represents the marginal cost of the generation supply. Therefore, based on a similar concept in [35], the generation supply cost of $i$ th PEV is supposed to $C_{g, i}=\frac{1}{2} a\left(\sum_{k=1}^{K_{i}} P_{E V, i}(k)\right)^{2}+$ $b\left(\sum_{k=1}^{K_{i}} P_{E V, i}(k)\right)+c$ with proper parameters $a, b$, and $c$. Therefore, a real-time price model for $i$ th PEV is adopted linearly with respect to its total demand during the charging duration, such that

$$
P_{r e, i}=a \sum_{k=1}^{K_{i}} P_{E V, i}(k)+b .
$$

Note that the real-time price model is widely applied for the coordination of PEV's charging process [36], [37].

Furthermore, the $i$ th PEV needs to reach its desired SoC, $\mu_{i}^{*}$ by its deadline that is determined by

$$
\sum_{k=1}^{K_{i}} P_{E V, i}(k) \Delta T_{i}=\left(\mu_{i}^{*}-\mu_{i}(0)\right) Q_{i} .
$$

By substituting (11) into (10), the price model is expressed as

$$
P_{r e, i}=a \frac{\left(\mu_{i}^{*}-\mu_{i}(0)\right) Q_{i}}{T_{i}}+b
$$

The satisfaction of PEV's customers from the charging service is usually dependent upon the SoC of their vehicles when finishing the charging process. Therefore, a concave 
utility function is defined to represent the satisfaction of $i$ th $\mathrm{PEV}$ customer, which relates to changing rate of SoC, such as

$$
\begin{aligned}
U_{E V, i}\left(\dot{\mu}_{i}\right)= & -\left(P_{r e, i} \frac{K_{i} \Delta T^{2}}{2}\right) \dot{\mu}_{i}{ }^{2} \\
& +\left(P_{r e, i}\left(\mu_{i}^{*}-\mu_{i}(0)\right) \Delta T\right) \dot{\mu}_{i} .
\end{aligned}
$$

It should note that the utility function has the following properties:

- The satisfaction of PEV's customers is increasing according to the changing rate of SoC.

- The utility function has the decreasing marginal utility.

The utility function has been widely applied in [36], [38][40] for PEV charging coordination problems. From the users' perspective, the utility function of PEV's users should be maximized when PEVs are connected to the charging station through a Smart Charger. The Smart Charger can regulate the charging current to maximize consumers' satisfaction. It is desired to discover an optimal charging current for each plugged PEV according its own utility function. Therefore, the optimal charging current reference for each PEV is formulated in following. By substituting (5) to (13), the utility function is rewritten as

$U_{E V, i}=-\left(P_{r e, i} \frac{K_{i} \Delta T^{2}}{2 Q_{i}^{2}}\right) I_{i}^{2}+\left(\frac{P_{r e, i}\left(\mu_{i}^{*}-\mu_{i}(0)\right) \Delta T}{Q_{i}}\right) I_{i}$

Then, by taking the derivative of (14) with respect to $I_{i}$ and equating to zero, the bliss point of the charging current of the utility function is

$$
I_{i}^{r e f}=\arg \max _{I_{i}}\left(U_{i}\left(\dot{\mu}_{i}\right)\right)=\frac{\mu_{i}^{*}-\mu_{i}(0)}{T_{i}} Q_{i},
$$

where $T_{i}=K_{i} \Delta T$.

To maximize the satisfaction of each PEV user, the PEV should be ideally charged following the desired charging current obtained in (15). However, due to the total available charging power constraints, it is almost impossible to realize the desired current charging for all PEVs. Therefore, inspired by a similar formulation process in [22], the objective function for PEVs is formulated by minimizing the difference between charging current and the desired reference, i.e., $f_{i}\left(P_{E V, i}\right)=$ $\epsilon_{i}\left(I_{i}^{r e f}-I_{i}\right)^{2}$. The total deviations are denoted by $f\left(P_{E V}\right)$ with $P_{E V}=\left[P_{E V, 1}, \ldots, P_{E V, n}\right]^{T} \in \mathbb{R}^{n}$. With (7), the objective function is written as

$$
\begin{aligned}
\operatorname{Min} f\left(P_{E V}\right)= & \operatorname{Min} \sum_{i=1}^{n} \epsilon_{i}\left(I_{i}^{r e f}-I_{i}\right)^{2} \\
& =\operatorname{Min} \sum_{i=1}^{n} \epsilon_{i}\left\{\left(I_{i}^{r e f}\right)^{2}+\frac{V_{o, i}^{2}}{2 R_{i}^{2}}+\frac{2 I_{i}^{r e f} V_{o, i}}{R_{i}}\right. \\
& \left.-\frac{V_{o, i}+2 R_{i} I_{i}^{r e f}}{2 R_{i}^{2}} \sqrt{4 R_{i} P_{E V, i}+V_{o, i}^{2}}+\frac{P_{E V, i}}{R_{i}}\right\},
\end{aligned}
$$

where $\epsilon_{i}$ is a non-negative weight given by

$$
\epsilon_{i}=\frac{1}{\mu_{i} T_{i}+\kappa} .
$$

This prior weight is introduced in terms of the current SoC and the total charging time of $i$ th PEV. A small positive value, $\kappa$, is set to avoid singularity. The weight can prioritize PEVs based on the time of charge and the remaining SoC to be charged.

The first three terms in (16) are independent of the decision variable $P_{E V, i}$, which can be neglected from the objective function. Hence (16) can be further simplified as

$$
\begin{gathered}
\operatorname{Min} \sum_{i=1}^{n} \epsilon_{i}\left(\frac{P_{E V, i}}{R_{i}}-\frac{V_{o, i}+2 R_{i} I_{i}^{r e f}}{2 R_{i}^{2}} \sqrt{4 R_{i} P_{E V, i}+V_{o, i}^{2}}\right) \\
\text { s.t. } \\
0 \leq P_{E V, i} \leq P_{E V, i}^{M},
\end{gathered}
$$

Furthermore, for the purpose of fully using the available power, we assume that

$$
\sum_{i=1}^{n} P_{E V, i}=P_{\text {total }} .
$$

The objective function (18) is convex, and the set of charging power allocations satisfying the box constrain is $\mathcal{F}_{\text {box }}=\left\{P_{E V, i} \in \mathbb{R} \mid 0 \leq P_{E V, i} \leq P_{E V, i}^{M}\right\}$. We denote the feasibility set of the above optimal problem as $\mathcal{F}_{E V}=$ $\left\{P_{E V, i} \in \mathbb{R} \mid 0 \leq P_{E V, i} \leq P_{E V, i}^{M}\right.$ and $\left.\mathbf{1}_{n}^{T} P_{E V}=P_{\text {total }}\right\}$. Besides, the solution is denoted by $\mathcal{F}_{E V}^{*}$, which is compact since $\mathcal{F}_{E V}$ is compact.

A centralized strategy can solve the above problem, but it requires a powerful control center to receive all the information of each PEV for data management, communication and processing. In this paper, our objective is to design a distributed cooperative control algorithm such that it can optimally allocate charging power of all PEVs based on their priorities. Furthermore, from any initial conditions, this novel charging control algorithm can accommodate plug-and-play operations and perform well under the time-varying supplydemand condition in an isolated power system.

\section{Distributed Optimal Solution}

In this section, a distributed control algorithm is proposed to solve the optimal charging control problem.

\section{A. Problem Reformulation}

The inequality constraint may cause difficulties in the optimal control design. Thus, the exact penalty function method is utilized to tackle this problem. The optimization problem (18) is reformulated by rewriting the objective function of $i$ th PEV, i.e.

$$
g_{i}\left(P_{E V, i}\right)=f_{i}\left(P_{E V, i}\right)+\frac{1}{\varepsilon}\left(\left[P_{E V, i}-P_{E V, i}^{M}\right]^{+}\right) .
$$

and $g\left(P_{E V}\right)=\sum_{i=1}^{n} g_{i}\left(P_{E V, i}\right)$, which subjects to the available charging power constraint $\mathbf{1}_{n}^{T} P_{E V}^{*}=P_{\text {total }}$.

Note that $g\left(P_{E V}\right)$ is convex, locally Lipschitz, and continuously differentiable on $\mathbb{R}$ except at $P_{E V, i}=P_{E V, i}^{M}$. According to the Proposition 5.2 in [23], the original optimization 
problem (18) and the reformulated optimal charging problem coincide if there exists $\varepsilon \in \mathbb{R}_{>0}$ such that

$$
\varepsilon<\frac{1}{2 \max _{P_{E V} \in \mathcal{F}_{E V}}\left\|\nabla g\left(P_{E V}\right)\right\|_{\infty}} .
$$

In our control design, we assume that (21) holds this condition.

A useful Lemma based on [41] is introduced as follows

Lemma 4.1: Since $g\left(P_{E V, i}\right)$ is convex, locally Lipschitz, and continuously differentiable except at $P_{E V, i}=P_{E V, i}^{M}$, the charging optimal problem has a solution $P_{E V}^{*} \in \mathbb{R}^{n}$ if and only if, there exists $\sigma \in \mathbb{R}$ such that

$$
\sigma \mathbf{1}_{n} \in \partial g\left(P_{E V}^{*}\right) \text { and } \mathbf{1}_{n}^{T} P_{E V}^{*}=P_{\text {total }} .
$$

\section{B. Distributed Algorithmic Design}

Inspired by the dynamic average consensus estimation method proposed in [42], a distributed optimal solution is presented to the optimal charging problem, which allows the power allocation of each PEV to start from any initial conditions. The optimal charging protocol is formulated as

$$
\begin{aligned}
\dot{P}_{E V, i} \in & -\sum_{j \in \mathbb{N}(i)} a_{i j}\left(\partial g_{i}\left(P_{E V, i}\right)-\partial g_{j}\left(P_{E V, j}\right)\right)+\gamma x_{i}, \\
\dot{x}_{i}= & -\beta\left(x_{i}-\left(b_{i 0} P_{\text {total }}-P_{E V, i}\right)\right) \\
& -\alpha \sum_{j \in \mathbb{N}(i)} a_{i j}\left(x_{i}-x_{j}\right)-\sum_{j \in \mathbb{N}(i)} a_{i j}\left(\eta_{i}-\eta_{j}\right), \\
\dot{\eta}_{i}= & \alpha \beta x_{i},
\end{aligned}
$$

where $\alpha, \beta, \gamma \in \mathbb{R}_{>0}$ are the parameters to be designed, $\mathbb{N}(i)$ denotes the neighbour set of $i$ th PEV. In (23a), the first term explores the minimization of the total cost, and a feedback element, $\gamma x_{i}$, enforces $i$ th PEV to satisfy the supplydemand equality condition. Furthermore, $x_{i}$ is designed to track the average signal $\frac{1}{n}\left(P_{\text {total }}-\mathbf{1}_{n}^{T} P_{E V}(t)\right)$ for each PEV $i \in\{1, \ldots, n\}$. Unlike [43], the proposed algorithm solves the optimal problem regardless of initial values of $\left(P_{0}, x_{0}, \eta_{0}\right)$, and as proved by [44], the proposed dynamic estimation method is a low-pass filter that ensure robust average consensus estimation in a sensor network.

\section{Convergence Analysis}

For convergence analysis, the algorithm is rewritten in a compact form represented by the set-valued map $\mathcal{X}_{E V}$

$$
\begin{aligned}
& \dot{P}_{E V} \in-\mathcal{L} \partial g\left(P_{E V}\right)+\gamma x, \\
& \dot{x}=-\alpha \mathcal{L} x-\beta\left(x-\left(B_{0} P_{\text {total }}-P_{E V}\right)\right)-\mathcal{L} \eta, \\
& \dot{\eta}=\alpha \beta x,
\end{aligned}
$$

where $x, \eta$ are the column vectors of $x_{i}$, and $\eta_{i}$ respectively.

We characterize the $\omega$-limit set of the trajectories of (24) with any initial conditions in $\mathbb{R}^{n} \times \mathbb{R}^{n} \times \mathbb{R}^{n}$.

Lemma 4.2: The $\omega$-limit set of the trajectories of (24) with any initial conditions in $\mathbb{R}^{n} \times \mathbb{R}^{n} \times \mathbb{R}^{n}$ is contained in $\mathcal{H}_{P_{\text {total }}} \times$ $\mathcal{H}_{0} \times \mathcal{H}_{0}$.
TABLE I

PARAMETERS OF PEV BATTERIES

\begin{tabular}{lcccccc}
\hline & $V_{o, i}(V)$ & $R_{i}(\mathrm{Ohm})$ & $Q_{i}(A . h)$ & $\mathrm{SoC}(0)$ & $S o C^{*}$ & $P_{E V, i}^{M}(k W)$ \\
\hline Vehicle 1 & 303 & 1.13 & 25 & 0.20 & 0.90 & 3.3 \\
Vehicle 2 & 292 & 1.08 & 30 & 0.24 & 0.85 & 3.3 \\
Vehicle 3 & 289 & 1.17 & 28 & 0.30 & 0.80 & 3.3 \\
Vehicle 4 & 301 & 1.12 & 29 & 0.18 & 0.85 & 3.3 \\
Vehicle 5 & 298 & 1.07 & 32 & 0.25 & 0.90 & 3.3 \\
Vehicle 6 & 306 & 1.14 & 35 & 0.21 & 0.90 & 3.3 \\
\hline
\end{tabular}

Proof: See appendix. B

Based on Lemma 4.2 and the Proposition A.1 in [43], we now ready to establish that, with (24), the trajectories of the charging power allocations of PEVs converge to the solution of the optimal charging problem.

Theorem 4.1: The trajectories of (24) converge to the solution of the optimal charging problem if $\alpha, \beta, \gamma \in \mathbb{R}_{>0}$ satisfy the condition that

$$
\frac{\gamma}{\alpha \beta \lambda_{2}\left(\mathcal{L}+\mathcal{L}^{T}\right)}+\frac{\beta \lambda_{\max }\left(\mathcal{L}^{T} \mathcal{L}\right)}{2}<\lambda_{2}\left(\mathcal{L}+\mathcal{L}^{T}\right) .
$$

Proof: See appendix. C

Remark 4.1: Assuming that each PEV is able to estimate the power losses of its adjacent transmission lines, the proposed solution could be extended to handle the transmission losses during the charging process of PEVs proposed in [45].

\section{Simulation RESUltS AND ANALYSis}

In the simulation studies, several cases are used to validate the effectiveness of the proposed distributed optimal strategy. The algorithm is tested for 5-PEV system on a PC with Inter(R) Core(TM) i7-4770 CPU @ 3.40GHz and 4GB RAM in MATLAB/Simulink. The parameters of the PEV battery are listed in Table I, which are taken from the typical battery charging profiles provided in [46]. The PEVs are assumed to be able to interact with their adjacent neighbours in the communication network. The total available charging power can be accessible by several PEVs with $\mathbf{1}_{n}^{T} B_{0}=1$. Without loss of generality, we assume that PEV1 knows the total available charging power.

\section{A. Algorithm Implementation}

The proposed optimal solution can be implemented in a MAS framework. The step-by-step algorithm for $i$ th PEV agent is shown in Algorithm. 1, and Fig. 2 gives the general operation structure of $i$ th agent. The communication topology for PEVs charging is shown as Fig. 3. Each PEV is plugged into the charging station through a recharging socket, which is assigned as an agent. The recharging socket only interacts with its neighboring agents to exchange the information, i.e., $\left(P_{E V, i}, x_{i}\right)$. Each agent deploys the proposed algorithm (24) in Section IV which will provide an optimal power charging reference for all PEVs.

Remark 5.1: The applicability of the proposed algorithm can be investigated by calculating the minimum amount data exchanged by each node. To this end, a simulation study is 
TABLE II

TOTAL AMOUNT OF DATA OF THE COMMUNICATION LINE BETWEEN PEV1 AND PEV2

\begin{tabular}{|c|c|c|c|c|}
\hline & Num of Variable & $\begin{array}{c}\text { bits } \\
\text { (Single-precision) }\end{array}$ & Sample Rate & Data \\
\hline$P_{1}$ & 1 & $32 \mathrm{bit}$ & $100 \mathrm{~Hz}$ & $3200 \mathrm{bps}$ \\
\hline$z_{1}$ & 1 & $32 \mathrm{bit}$ & $100 \mathrm{~Hz}$ & $3200 \mathrm{bps}$ \\
\hline$\eta_{1}$ & 1 & $32 \mathrm{bit}$ & $100 \mathrm{~Hz}$ & $3200 \mathrm{bps}$ \\
\hline \multicolumn{3}{|c|}{ Total amount of data } & $9600 \mathrm{bps}$ \\
\hline
\end{tabular}

provided to show the minimum amount of data exchanged by each node on the communication network. In our simulation study, the communication network is designed as a directed graph, and the communication channel between each PEV has the same tolerance for the data transmission. Without loss of generality, we calculated the date exchanged in the communication channel between PEV1 and PEV2. The sample time is set $0.01 \mathrm{~s}(100 \mathrm{~Hz})$. Our algorithm has 3 states for each agent that requires 3 real numbers. The amount of data of one directed communication channel from PEV1 to PEV2 can be calculated as Table II. As the result shown, the minimum amount of data of the communication channel from PEV1 to PEV2 is 9.6Kbps.

Remark 5.2: The results of our simulation studies are obtained based on the rigorous parameter selection, which is taken from a standard charging profile, and the constraint of the charging rate is set based on the Level 2 charging profile. As a result, the proposed algorithm is well-adapted to a real PEV charging problem. Furthermore, as the Remark 5.1 shown, the minimal amount of data exchanged by each PEV may be compatible with the throughput of modern communication systems. With the above analysis, it is shown that the proposed algorithm is potentially implemented in a testbed.

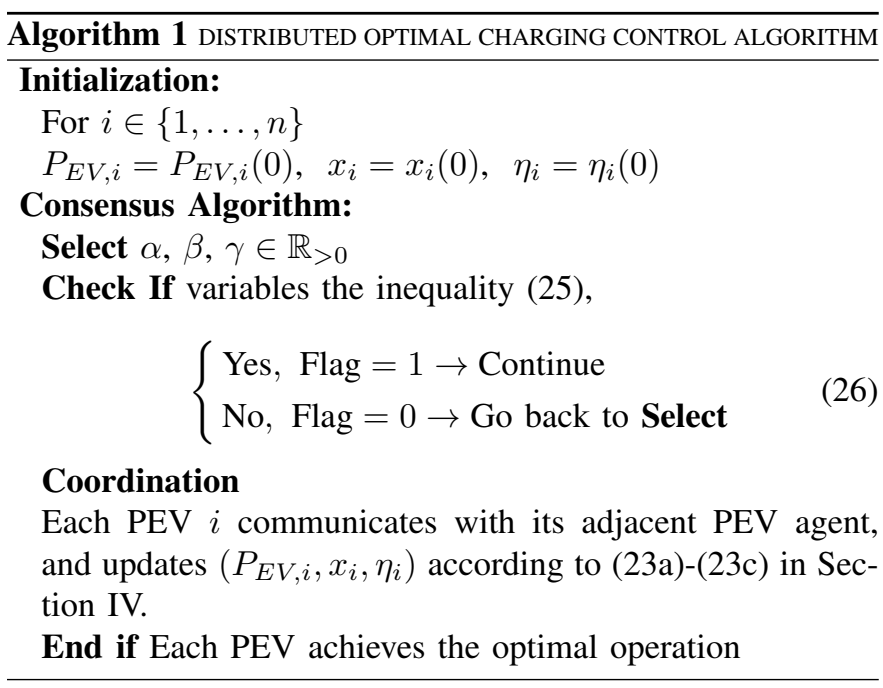

\section{B. Simulation Studies}

In the case studies, the designed parameters are chosen as $\alpha=12, \beta=0.4, \gamma=2, \varepsilon=0.0085$, which satisfy the condition in (21), and (25) specified in Theorem 4.1. In Case 5.1, we study the optimal charging problem with the constant total

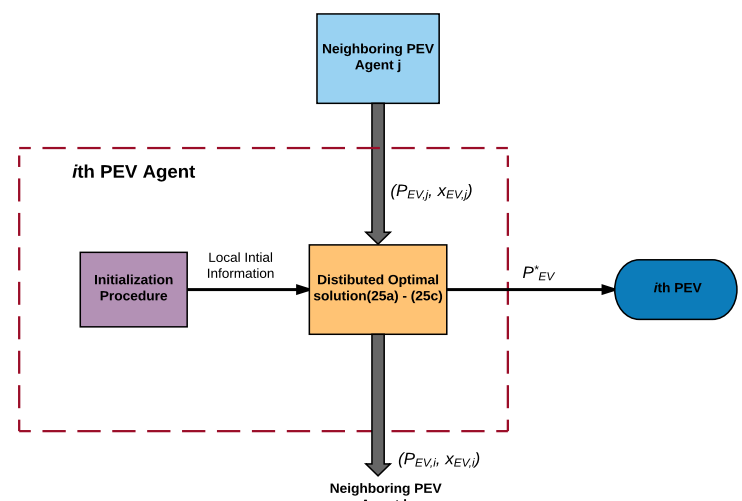

Fig. 2. The general operation structure of $i$ th agent

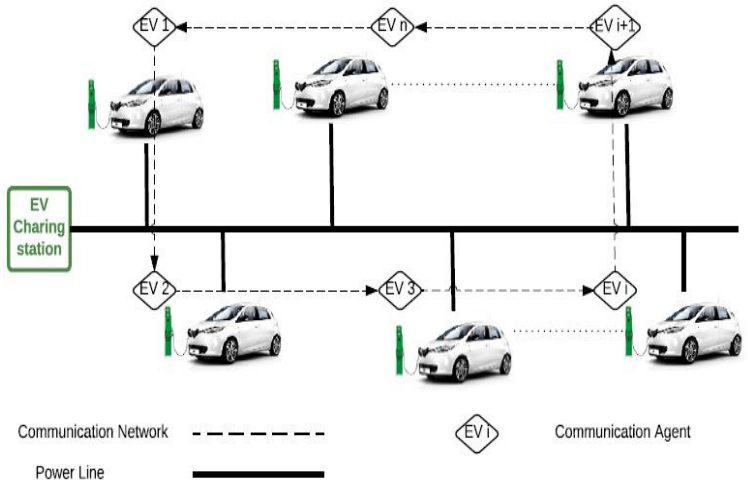

Fig. 3. The communication topology for PEVs charging

available charging power while the plug-and-play operation is considered. Case 5.2 investigates the performance of the proposed strategy under the time-varying available charging power supply. Finally, the scalability analysis is validated in Case 5.3.

Case 5.1:

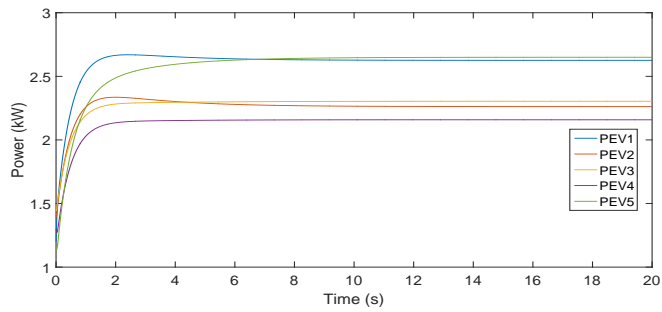

Fig. 4. The charging power updates for PEVs

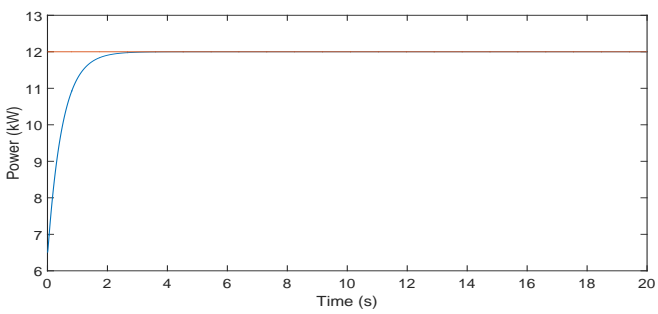

Fig. 5. Total allocated charging power updates for PEVs 


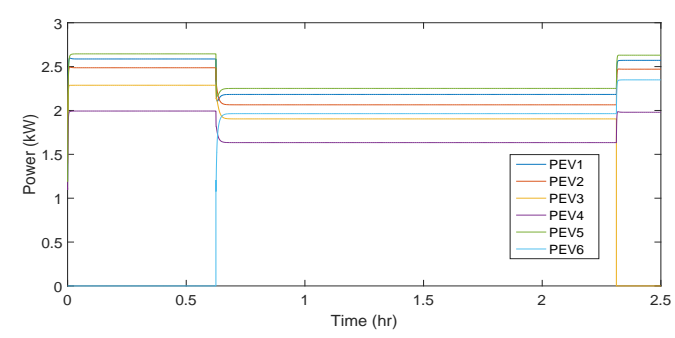

Fig. 6. Allocated charging power updates for PEVs

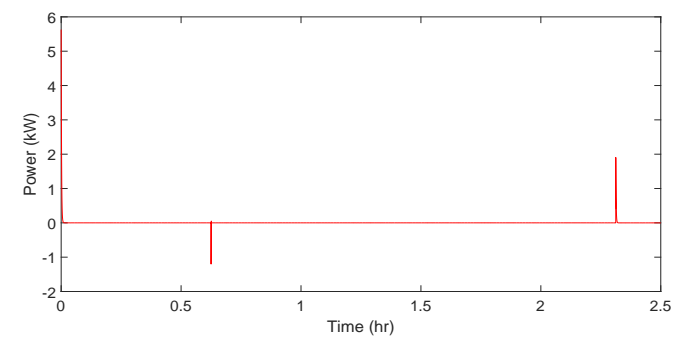

Fig. 7. Supply-demand mismatch updates for PEVs

In this case, the total available charging power is assumed as $12 \mathrm{~kW}$. As shown in Figs. $4-5$, the charging rates of all PEVs quickly converge to their optimal values, while the total allocated charging power converges to $12 \mathrm{~kW}$, i.e., the total available charging power.

Now, the plug-and-play adaptability of our strategy is investigated, e.g., 1) an EV arrives in the charging station at an arbitrary time; 2) an EV departs from the charging station when its $\mathrm{SoC}$ is charged to its desired value. The total available charging power is still $12 \mathrm{~kW}$. The communication network is weight-balanced, when PEVs are moving in and out. Here an imbalance-correcting algorithm [47] is applied to our communication network design. Supposed that each agent can correct its weight through sending and receiving information from its neighbours such that the digraph adapts to be weightbalanced in a finite time. Figs. $6-8$ show the charging power allocations of PEVs during their charging process, the demandsupply mismatch and the SoC of PEVs. As shown in Fig. 6, the charging power allocations of each PEV converge to their optimal values. When one PEV, e.g., PEV6, arriving in the charging station, the charging power allocation can converge to the optimal values with the proposed strategy. After PEV3 departing from the charging station, the proposed strategy guarantees that the rest of PEVs can still reach their optimal

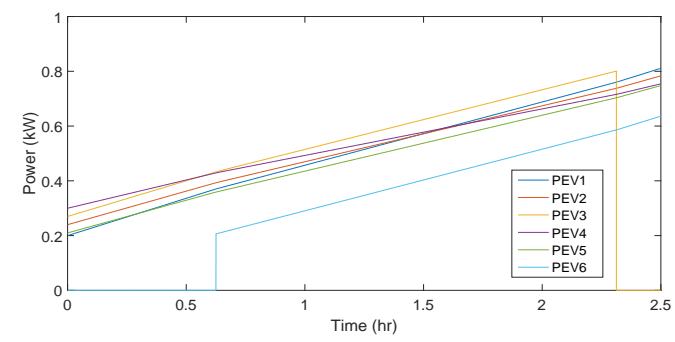

Fig. 8. The SoC updates for PEVs

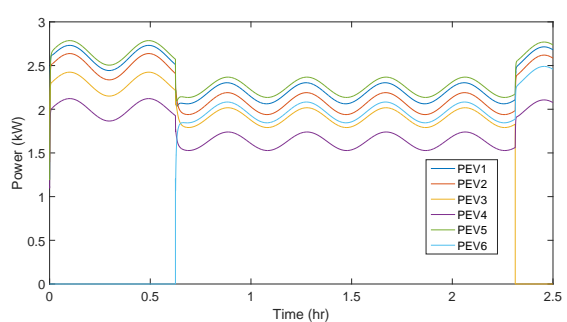

Fig. 9. Allocated charging power updates for PEVs

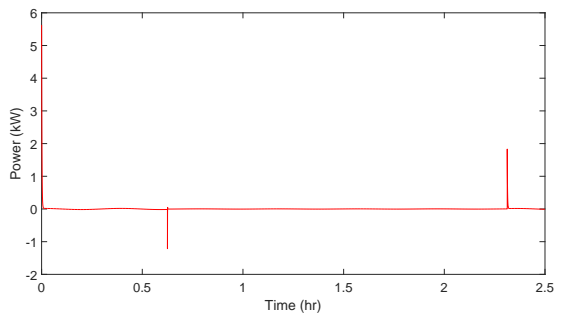

Fig. 10. Supply-demand mismatch updates for PEVs

charging rates by sharing the total available charging power. As shown in Fig. 7, during the whole PEV charging process, the deviations between the total available charging power and the total allocated charging power are very small. Fig. 8 gives the SoC update of PEVs charging process when PEV departing from or arriving at the charging station. When PEV6 arriving at the charging station at a random time, its $\mathrm{SoC}$ starts from $20 \%$ and increases during charging process. The SoC of PEV3 drops to zero because it has been charged to the desired SoC, and it is ready to depart from the station. Therefore, the proposed strategy will have little effect on the frequency disturbance of the system, which may be applied to isolated systems such as an autonomous microgrid.

Case 5.2:

The effectiveness of the proposed strategy under a timevarying supply-demand condition is validated in this case. An isolated microgrid consisting of distributed generators (DGs) and loads is considered here. Due to the intermittent of renewable energy sources, particularly the wind power generation, the generated power may fluctuate and cause the frequency fluctuation problem in the microgrid. PEVs that installed in the customer side are employed as a flexible load for alleviate frequency fluctuation [48]. To this end, we consider a time-varying non-PEV load condition with the total available power given by $P_{\text {total }}=12000+700 \sin (0.005 t)$. The communication network and the other operating conditions are the same as those in Case 5.1. As shown in Figs. 9 - 10, the allocated charging powers converge to their optimal values under the time-varying supply-demand condition, while the mismatch between supply and demand power converges to zero. In addition, Fig. 10 shows the proposed strategy effectively offsets the supply-demand mismatch, which will help with frequency fluctuation (caused by intermittent renewable sources) regulation.

Case 5.3:

The scalability of the proposed strategy is validated. To do 


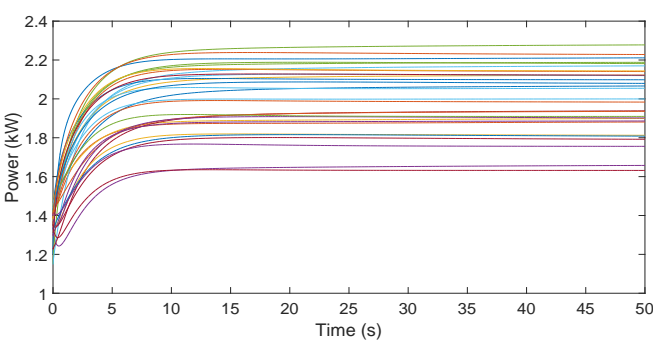

Fig. 11. Allocated charging power updates for 30-PEVs

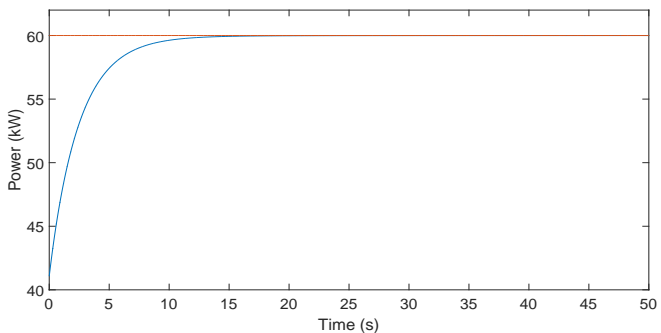

Fig. 12. Supply-demand mismatch updates for 30-PEVs

so, we implement the optimal control strategy to both 30-PEV system and 60-PEV system, and The total available charging power is supposed to be $60 \mathrm{~kW}$ and $120 \mathrm{~kW}$, respectively. The communication network is weight-balanced and strongly connected.

As shown in Fig. 11 and Fig. 13, the proposed strategy can guarantee the allocated charging powers to converge to their optimal values within 25s. Figs. 12 - 14 illustrate the deviations of demand and supply power can converge to zero. It is worth noting that the convergence of the proposed algorithm is mainly determined by the parameter selection and the knowledge of the communication network. The convergence can be ensured for a large number fleet by maintaining the

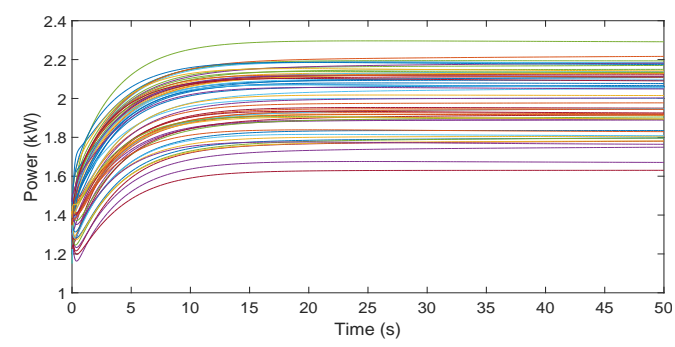

Fig. 13. Allocated charging power updates for 60-PEVs

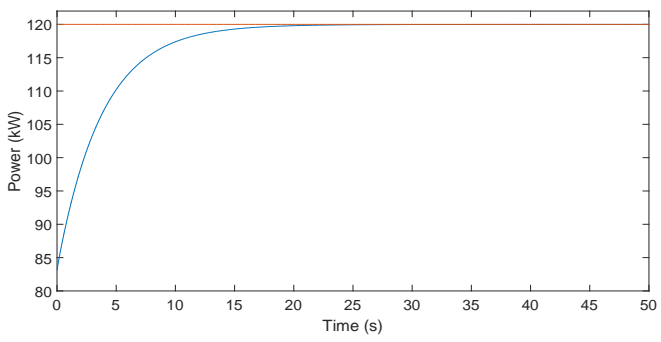

Fig. 14. Supply-demand mismatch updates for 60-PEVs inequality (25).

It should be noted that the proposed algorithm is applicable in a real scenario since the required communication network for data transmission is acceptable for a real communication network and the computational cost is acceptable for an embedded system running.

\section{CONCLUSION}

In this paper, a cooperative distributed control strategy is proposed for PEVs optimal charging by maximizing the welfare and satisfaction of PEV customers while considering the PEVs' charging constraints. The proposed distributed algorithm is implemented based on MAS-framework under a directed communication graph, which is robust to singlelink failures compared with the centralized methods. The initializing procedures are no longer needed in our control design. Thus, the PEVs can start from any charging power allocations. In addition, its convergence does not rely on the specific graph, which admits the plug-and-play operation during PEV charging process. Furthermore, the proposed distributed strategy can handle the time-varying supply-demand mismatch problem in isolated systems.

\section{APPENDIX}

\section{A. Nonsmooth Analysis and Differential Inclusions}

For any $\varepsilon \in(0, \infty)$, a set-valued map $\mathcal{H}: \mathbb{R}^{n} \rightrightarrows \mathbb{R}^{m}$ is said upper semi-continuous at $x \in \mathbb{R}^{n}$ if there exists $\delta \in(0, \infty)$ such that $\mathcal{H}(y) \subset \mathcal{H}(x)+B(0, \varepsilon)$ for all $y \in B(x, \delta)$. Also, $\mathcal{H}$ is locally bounded at $x \in \Re^{n}$ if there exist $\varepsilon, \delta \in(0, \infty)$ such that $\|z\| \leq \varepsilon$ for all $z \in \mathcal{H}(y)$ and $y \in B(x, \delta)$. Let $\Omega_{f}$ be the set of points where $f$ is not differentiable, the generalized gradient of $f$ is defined as

$$
\partial f(x)=\operatorname{co}\left\{\lim _{k \rightarrow \infty} \nabla f\left(x_{k}\right) \mid x_{k} \rightarrow x, x_{k} \notin \Omega_{f} \cup S\right\}
$$

where co denotes convex hull and $S$ is a set of measure zero.

A solution of $\dot{x} \in \mathcal{H}(x)$ on $[0, T] \subset \mathbb{R}$ is defined as an absolutely continuous map $x:[0, T] \rightarrow \mathbb{R}^{n}$ that satisfies (2) for almost all $t \in[0, T]$. Also, if $\mathcal{H}$ is locally bounded, upper semi-continuous, and takes non-empty, compact, and convex values, then the existence of solutions is guaranteed.

\section{B. Proof of Lemma 4.2}

Proof: Defining $\psi(t)=\mathbf{1}_{n}^{T} P_{E V}(t)-P_{\text {total }}$, one has

$$
\dot{\psi}(t)=\mathbf{1}_{n}^{T} \dot{P}_{E V}(t)=\mathbf{1}_{n}^{T} \gamma x(t),
$$

and

$$
\begin{aligned}
\ddot{\psi}(t) & =\mathbf{1}_{n}^{T} \gamma \dot{x}(t) \\
& =-\mathbf{1}_{n}^{T} \beta \gamma x(t)+\mathbf{1}_{n}^{T} \beta \gamma\left(B_{0} P_{\text {total }}-P_{E V}(t)\right) \\
& =-\beta \gamma \psi(t)-\beta \dot{\psi}(t) .
\end{aligned}
$$

The system can be rewritten as

$$
\dot{z}=A z
$$


where $z=\left[z_{1}, z_{2}\right]^{T}$, and $z_{1}=\psi, z_{2}=\dot{\psi}$. The system matrix, $A$, is obtained as

$$
A=\left[\begin{array}{cc}
0 & 1 \\
-\beta \gamma & -\beta
\end{array}\right]
$$

Let $M \in \mathbb{R}^{2 \times 2}$ be

$$
M=\frac{1}{2 \gamma \beta^{2}}\left[\begin{array}{cc}
\beta^{2}+\beta \gamma+(\beta \gamma)^{2} & \beta \\
\beta & 1+\beta \gamma
\end{array}\right],
$$

which satisfies $A^{T} M+M A+I=0$. Define $V_{z}=z^{T} M z$ as a Lyapunov function candidate for (29), and the derivative of $V_{z}$ is

$$
\dot{V}_{z}=-z^{T} z .
$$

Therefore, we can deduce that $\lim _{t \rightarrow \infty} z_{i}(t)=0$ for $i=1,2$, and the convergence rate is exponential. Furthermore, $z_{i}(t)=$ 0 implies that $\mathbf{1}_{n}^{T} P_{E V}(t) \rightarrow P_{\text {total }}$ and $\mathbf{1}_{n}^{T} x(t) \rightarrow 0$. Note that $\mathbf{1}_{n}^{T} \dot{\eta}=0$, as $\mathbf{1}_{n}^{T} x(t) \rightarrow 0$.

\section{Proof of Theorem 4.1}

Proof: A change of coordinates is introduced to shift the equilibrium point of (24) to the origin. With $\bar{\eta}=\mathcal{L} \eta-$ $\beta\left(B_{0} P_{\text {total }}-P_{E V}\right)$, the set-valued map $\mathcal{X}_{E V}$ is transformed as

$$
\begin{gathered}
\mathcal{X}_{E V}\left(P_{E V}, x, \eta\right)=\{[-\mathcal{L} \xi+\gamma x,-(\alpha \mathcal{L}+\beta I) x-\bar{\eta}, \\
\left.(\alpha \beta \mathcal{L}+\gamma \beta I) x-\beta \mathcal{L} \xi]^{T} \in \mathbb{R}^{3 n} \mid \xi \in \partial g\left(P_{E V}\right)\right\} .
\end{gathered}
$$

Consider a candidate Lyapunov function $V_{2}: \mathbb{R}^{3 n} \rightarrow \mathbb{R}_{\geq 0}$,

$$
V_{2}=g\left(P_{E V}\right)+\frac{1}{2} \gamma \beta\|x\|^{2}+\frac{1}{2}\|\beta x+\bar{\eta}\|^{2},
$$

and let $\varphi_{1}=x, \varphi_{2}=\beta x+\bar{\eta}$, then

$$
V_{2}=g\left(P_{E V}\right)+\frac{1}{2} \gamma \beta\left\|\varphi_{1}\right\|^{2}+\frac{1}{2}\left\|\varphi_{2}\right\|^{2} .
$$

Define the overall coordinate transformation $\mathcal{T}: \mathbb{R}^{3 n} \rightarrow \mathbb{R}^{3 n}$ as

$$
\begin{aligned}
{\left[P_{E V}, \varphi_{1}, \varphi_{2}\right]^{T} } & =\mathcal{T}\left(P_{E V}, x, \eta\right) \\
& =\left[P_{E V}, x, \beta x+\mathcal{L} \eta-\beta\left(B_{0} P_{\text {total }}-P_{E V}\right)\right]^{T}
\end{aligned}
$$

Next step is to prove that, in the new coordinate, the trajectories of (24) converge to the set

$$
\overline{\mathcal{F}}_{\text {op }}=\mathcal{T}\left(\mathcal{F}_{o p}^{*}\right)=\mathcal{F}_{E V}^{*} \times\{0\} \times\{0\} .
$$

Note that $g$ is locally Lipschitz and regular, while the setvalued map $\mathcal{X}_{E V}$ is locally bounded, upper semi-continuous, and takes non-empty, compact and convex values. Take the setvalued Lie derivative $L_{\mathcal{X}_{E V}} V_{2}: \mathbb{R}^{n} \rightrightarrows \mathbb{R}$ of $V_{2}\left(P_{E V}, \varphi_{1}, \varphi_{2}\right)$ along the $\mathcal{X}_{E V}$,

$$
\begin{aligned}
L_{\mathcal{X}_{E V}} V_{2} & =\left\{-\xi^{T} \mathcal{L} \xi+\gamma \xi^{T} \varphi_{1}-\gamma \alpha \beta \varphi_{1}^{T} \mathcal{L} \varphi_{1}\right. \\
& \left.-\beta\left\|\varphi_{2}\right\|^{2}-\beta \varphi_{2}^{T} \mathcal{L} \xi \mid \xi \in \partial g\left(P_{E V}\right)\right\} .
\end{aligned}
$$

Denote $\delta=\left[\delta_{1}, \delta_{2}, \delta_{3}\right]^{T}$, where $\delta_{1}=\xi \in \partial g\left(P_{E V}\right), \delta_{2}=$ $\beta \gamma \varphi_{1}$ and $\delta_{3}=\varphi_{2}$ respectively. A continuous function is defined as $w: \mathbb{R}^{3 n} \times \mathbb{R}^{3 n} \rightarrow \mathbb{R}^{3 n}$,

$$
\begin{aligned}
w\left(P_{E V}, \varphi_{1}, \varphi_{2}, \delta\right)= & {\left[-\mathcal{L} \delta_{1}+\gamma \varphi_{1},-\alpha \mathcal{L} \varphi_{1}-\varphi_{2},\right.} \\
& \left.\beta \gamma \varphi_{1}-\beta \varphi_{2}-\beta \mathcal{L} \delta_{1}\right]^{T},
\end{aligned}
$$

and hence dynamics (32) can be expressed as

$$
\begin{aligned}
\mathcal{X}_{E V}\left(P_{E V}, \varphi_{1}, \varphi_{2}\right)= & \left\{w\left(P_{E V}, \varphi_{1}, \varphi_{2}, \delta\right) \mid\right. \\
& \left.\delta \in \partial V_{2}\left(P_{E V}, \varphi_{1}, \varphi_{2}\right)\right\} .
\end{aligned}
$$

Since the directed graph $\mathcal{G}$ is strongly connected and weightbalanced with the fact that $\mathbf{1}_{n}^{T} \varphi_{1}=0$ for $\left(P_{E V}, \varphi_{1}, \varphi_{2}\right) \in$ $\mathcal{H}_{P_{\text {total }}} \times \mathcal{H}_{0} \times \mathcal{H}_{0}$,

$$
\begin{aligned}
& \delta^{T} w\left(P_{E V}, \varphi_{1}, \varphi_{2}, \delta\right)=-\frac{1}{2} \xi^{T}\left(\mathcal{L}+\mathcal{L}^{T}\right) \xi+\gamma \xi^{T} \varphi_{1} \\
& \quad-\frac{1}{2} \alpha \beta \gamma \varphi_{1}^{T}\left(\mathcal{L}+\mathcal{L}^{T}\right) \varphi_{1}-\beta\left\|\varphi_{2}\right\|^{2}-\beta \varphi_{2}^{T} \mathcal{L} \xi \\
& \quad \leq-\frac{1}{2} \lambda_{2}\left(\mathcal{L}+\mathcal{L}^{T}\right)\left\|\xi-\frac{1}{n} \mathbf{1}_{n}^{T} \mathbf{1}_{n} \xi\right\|^{2} \\
& \quad+\gamma\left(\xi-\frac{1}{n} \mathbf{1}_{n}^{T} \mathbf{1}_{n} \xi\right)^{T} \varphi_{1}-\frac{1}{2} \alpha \beta \gamma \lambda_{2}\left(\mathcal{L}+\mathcal{L}^{T}\right)\left\|\varphi_{1}\right\|^{2} \\
& \quad-\beta\left\|\varphi_{2}\right\|^{2}-\beta \varphi_{2}^{T} \mathcal{L}\left(\xi-\frac{1}{n} \mathbf{1}_{n}^{T} \mathbf{1}_{n} \xi\right)
\end{aligned}
$$

Defining $\vartheta=\xi-\frac{1}{n}\left(\mathbf{1}_{n}^{T} \mathbf{1}_{n} \xi\right)$, and $\phi^{T}=\left[\vartheta^{T}, \varphi_{1}^{T}, \varphi_{2}^{T}\right]$, we have

$$
\delta^{T} w\left(P_{E V}, \varphi_{1}, \varphi_{2}, \delta\right) \leq \phi^{T} R \phi
$$

where

$R=\left[\begin{array}{ccc}-\frac{1}{2} \lambda_{2}\left(\mathcal{L}+\mathcal{L}^{T}\right) I & \frac{1}{2} \gamma I & -\frac{1}{2} \beta \mathcal{L}^{T} \\ \frac{1}{2} \gamma I & -\frac{1}{2} \alpha \beta \gamma \lambda_{2}\left(\mathcal{L}+\mathcal{L}^{T}\right) I & 0 \\ -\frac{1}{2} \beta \mathcal{L}^{T} & 0 & -\beta I\end{array}\right]$.

Applying the Schur complement, $R \in \mathbb{R}^{3 n \times 3 n}$ is negative definite if

$$
\begin{aligned}
& -\frac{1}{2} \lambda_{2}\left(\mathcal{L}+\mathcal{L}^{T}\right) I \\
& -\left[\begin{array}{ll}
\frac{1}{2} \gamma I & -\frac{1}{2} \beta \mathcal{L}^{T}
\end{array}\right]\left[\begin{array}{cc}
-\frac{1}{2} \alpha \beta \gamma \lambda_{2}\left(\mathcal{L}+\mathcal{L}^{T}\right) I & 0 \\
0 & -\beta I
\end{array}\right]^{-1}\left[\begin{array}{c}
\frac{1}{2} \gamma I \\
-\frac{1}{2} \beta \mathcal{L}
\end{array}\right] \\
& =-\frac{1}{2} \lambda_{2}\left(\mathcal{L}+\mathcal{L}^{T}\right) I+\frac{\gamma}{2 \alpha \beta \lambda_{2}\left(\mathcal{L}+\mathcal{L}^{T}\right)} I+\frac{\beta}{4} \mathcal{L}^{T} \mathcal{L},
\end{aligned}
$$

is negative definite, which is guaranteed by (25). Hence, $\delta^{T} w\left(P_{E V}, \varphi_{1}, \varphi_{2}, \delta\right) \leq 0$, and $\delta^{T} w\left(P_{E V}, \varphi_{1}, \varphi_{2}, \delta\right)=0$ if and only if $\phi=\varphi_{1}=\varphi_{2}=0$. Reasoning with Lemma A.1 [43], we conclude that $0 \in L_{\mathcal{X}_{E V}} V_{2}$ if and only if there exists $\sigma \in \mathbb{R}$ such that $\sigma \mathbf{1}_{n} \in \partial g\left(P_{E V}^{*}\right)$. With Lemma 4.1, $P_{E V}^{*} \in \mathcal{F}_{E V}$ being a solution of the optimal charging problem.

The last step is to show the trajectories of $\mathcal{T}$ are bounded. This follows similar lines in [43], and therefore we omit it here due to the page limitation. 


\section{REFERENCES}

[1] R. C. Green, L. Wang, and M. Alam, "The impact of plug-in hybrid electric vehicles on distribution networks: A review and outlook," Renew. Sustain. Energy Rev., vol. 15, no. 1, pp. 544-553, 2011.

[2] W. Su, H. Eichi, W. Zeng, and M. Y. Chow, "A survey on the electrification of transportation in a smart grid environment," IEEE Trans. Ind. Informat., vol. 8, no. 1, pp. 1-10, Feb 2012.

[3] J. Wang, C. Liu, D. Ton, Y. Zhou, J. Kim, and A. Vyas, "Impact of plugin hybrid electric vehicles on power systems with demand response and wind power," Energy Policy, vol. 39, no. 7, pp. 4016-4021, 2011.

[4] J. Pahasa and I. Ngamroo, "PHEVs bidirectional charging/discharging and soc control for microgrid frequency stabilization using multiple mpc," IEEE Trans. Smart Grid, vol. 6, no. 2, pp. 526-533, 2015.

[5] E. Sortomme and M. A. El-Sharkawi, "Optimal charging strategies for unidirectional vehicle-to-grid," IEEE Trans. Smart Grid, vol. 2, no. 1, pp. 131-138, 2011

[6] M. Yilmaz and P. T. Krein, "Review of the impact of vehicle-to-grid technologies on distribution systems and utility interfaces," IEEE Trans. Power Electron., vol. 28, no. 12, pp. 5673-5689, 2013.

[7] W. Su, H. Eichi, W. Zeng, and M.-Y. Chow, "A survey on the electrification of transportation in a smart grid environment," IEEE Trans. Ind. Informat., vol. 8, no. 1, pp. 1-10, 2012.

[8] D. Wu, D. C. Aliprantis, and L. Ying, "Load scheduling and dispatch for aggregators of plug-in electric vehicles," IEEE Trans. Smart Grid, vol. 3, no. 1, pp. 368-376, 2012.

[9] O. Sundström and C. Binding, "Optimization methods to plan the charging of electric vehicle fleets," in Proc. Int. Conf. Commun. Power Eng., 2010, pp. 28-29.

[10] F. Kennel, D. Gorges, and S. Liu, "Energy management for smart grids with electric vehicles based on hierarchical mpc," IEEE Trans. Ind. Informat., vol. 9, no. 3, pp. 1528-1537, 2013.

[11] N. Rotering and M. Ilic, "Optimal charge control of plug-in hybrid electric vehicles in deregulated electricity markets," IEEE Trans. Power Syst., vol. 26, no. 3, pp. 1021-1029, 2011.

[12] V. Moghadam, R. Mohammad, R. Zhang, and R. T. Ma, "Demand response for contingency management via real-time pricing in smart grids," in Smart Grid Commun.(SmartGridComm), 2014 IEEE Int. Conf. IEEE, 2014, pp. 632-637.

[13] C. Liu, K. Chau, D. Wu, and S. Gao, "Opportunities and challenges of vehicle-to-home, vehicle-to-vehicle, and vehicle-to-grid technologies," Proc. IEEE, vol. 101, no. 11, pp. 2409-2427, 2013.

[14] C.-K. Wen, J.-C. Chen, J.-H. Teng, and P. Ting, "Decentralized plug-in electric vehicle charging selection algorithm in power systems," IEEE Trans. Power Syst., vol. 3, no. 4, pp. 1779-1789, 2012.

[15] I. G. Unda, P. Papadopoulos, S. Skarvelis-Kazakos, L. M. Cipcigan, N. Jenkins, and E. Zabala, "Management of electric vehicle battery charging in distribution networks with multi-agent systems," Electric Power Systems Research, vol. 110, pp. 172-179, 2014.

[16] Y. Ota, H. Taniguchi, T. Nakajima, K. M. Liyanage, J. Baba, and A. Yokoyama, "Autonomous distributed V2G (vehicle-to-grid) satisfying scheduled charging," IEEE Trans. Smart Grid, vol. 3, no. 1, pp. 559-564, 2012.

[17] P. Papadopoulos, N. Jenkins, L. M. Cipcigan, I. Grau, and E. Zabala, "Coordination of the charging of electric vehicles using a multi-agent system," IEEE Trans. Smart Grid, vol. 4, no. 4, pp. 1802-1809, 2013.

[18] K. Kok, B. Roossien, P. MacDougall, O. van Pruissen, G. Venekamp, R. Kamphuis, J. Laarakkers, and C. Warmer, "Dynamic pricing by scalable energy management systemsfield experiences and simulation results using powermatcher," in Proc. 2012 IEEE Power and Energy Society General Meeting,. IEEE, 2012, pp. 1-8.

[19] G. Mokhtari, G. Nourbakhsh, and A. Ghosh, "Smart coordination of energy storage units (esus) for voltage and loading management in distribution networks," IEEE Trans. Power Syst., vol. 28, no. 4, pp. 4812-4820, 2013

[20] W.-J. Ma, V. Gupta, and U. Topcu, "On distributed charging control of electric vehicles with power network capacity constraints," in American Control Conf. (ACC), 2014. IEEE, 2014, pp. 4306-4311.

[21] N. Rahbari-Asr and M.-Y. Chow, "Cooperative distributed demand management for community charging of phev/pevs based on KKT conditions and consensus networks," IEEE Trans. Ind. Informat., vol. 10, no. 3, pp. 1907-1916, 2014.

[22] Y. Xu, "Optimal distributed charging rate control of plug-in electric vehicles for demand management," IEEE Trans. Power Syst., vol. 30 no. 3, pp. 1536-1545, 2015.

[23] A. Cherukuri and J. Cortés, "Distributed generator coordination for initialization and anytime optimization in economic dispatch," IEEE Trans. Control Syst. Technol., vol. 2, no. 3, pp. 226-237, 2015.
[24] Z. Ding, "Distributed adaptive consensus output regulation of networkconnected heterogeneous unknown linear systems on directed graphs," IEEE Trans. Autom. Control, vol. PP, no. 99, pp. 1-1, 2016.

[25] F. H. Clarke, Optimization and nonsmooth analysis. Siam, 1990, vol. 5.

[26] J. Cortes, "Discontinuous dynamical systems," IEEE Control Syst., vol. 28, no. 3, pp. 36-73, 2008.

[27] M. Ceraolo, "New dynamical models of lead-acid batteries," IEEE Trans. Power Syst., vol. 15, no. 4, pp. 1184-1190, 2000.

[28] X. Hu, S. Li, and H. Peng, "A comparative study of equivalent circuit models for li-ion batteries," J. Power Sources, vol. 198, pp. 359-367, 2012.

[29] P. Ramadass, B. Haran, P. M. Gomadam, R. White, and B. N. Popov, "Development of first principles capacity fade model for li-ion cells," $J$. The Electrochemical Soc., vol. 151, no. 2, pp. A196-A203, 2004.

[30] V. Johnson, "Battery performance models in advisor," J. Power Sources, vol. 110, no. 2, pp. 321-329, 2002.

[31] Y. Hu, S. Yurkovich, Y. Guezennec, and R. Bornatico, "Model-based calibration for battery characterization in hev applications," in 2008 American Control Conference. IEEE, 2008, pp. 318-325.

[32] S. Stockar, V. Marano, G. Rizzoni, and L. Guzzella, "Optimal control for plug-in hybrid electric vehicle applications," in Proceedings of the 2010 American control conference. IEEE, 2010, pp. 5024-5030.

[33] S. Bashash and H. K. Fathy, "Cost-optimal charging of plug-in hybrid electric vehicles under time-varying electricity price signals," IEEE Trans. Intell. Transp. Syst., vol. 15, no. 5, pp. 1958-1968, 2014.

[34] Z. Ma, S. Zou, and X. Liu, "A distributed charging coordination for large-scale plug-in electric vehicles considering battery degradation cost," IEEE Trans. Control Syst. Technol., vol. 23, no. 5, pp. 2044-2052, Sept 2015

[35] Z. Fan, "A distributed demand response algorithm and its application to phev charging in smart grids," IEEE Trans. on Smart Grid, vol. 3, no. 3, pp. 1280-1290, Sept 2012

[36] N. Rahbari-Asr, M. Y. Chow, Z. Yang, and J. Chen, "Network cooperative distributed pricing control system for large-scale optimal charging of PHEVs/PEVs," in Industrial Electronics Society, IECON 2013 - 39th Annual Conference of the IEEE, Nov 2013, pp. 6148-6153.

[37] Z. Ma, D. S. Callaway, and I. A. Hiskens, "Decentralized charging control of large populations of plug-in electric vehicles," IEEE Trans. Control Syst. Technol., vol. 21, no. 1, pp. 67-78, 2013.

[38] Z. Fan, "A distributed demand response algorithm and its application to phev charging in smart grids," IEEE Trans. Smart Grid, vol. 3, no. 3, pp. 1280-1290, 2012

[39] L. Gan, U. Topcu, and S. H. Low, "Optimal decentralized protocol for electric vehicle charging," IEEE Trans. Power Syst., vol. 28, no. 2, pp. 940-951, May 2013.

[40] N. Rahbari-Asr, M. Y. Chow, J. Chen, and R. Deng, "Distributed realtime pricing control for large scale unidirectional V2G with multiple energy suppliers," IEEE Trans. Ind. Informat., vol. PP, no. 99, pp. 1-1, 2016.

[41] D. P. Bertsekas, "Necessary and sufficient conditions for a penalty method to be exact," Math. Programming, vol. 9, no. 1, pp. 87-99, 1975.

[42] S. Li and Y. Guo, "Dynamic consensus estimation of weighted average on directed graphs," Int. J. Syst. Sci., vol. 46, no. 10, pp. 1839-1853, 2015.

[43] A. Cherukuri and J. Cortes, "Initialization-free distributed coordination for economic dispatch under varying loads and generator commitment," arXiv preprint arXiv:1409.4382, 2014.

[44] H. Bai, R. A. Freeman, and K. M. Lynch, "Robust dynamic average consensus of time-varying inputs," in 49th IEEE Conference on Decision and Control (CDC), Dec 2010, pp. 3104-3109.

[45] E. Sortomme, M. M. Hindi, S. J. MacPherson, and S. Venkata, "Coordinated charging of plug-in hybrid electric vehicles to minimize distribution system losses," IEEE Trans. Smart Grid, vol. 2, no. 1, pp. 198-205, 2011

[46] K. Morrow, D. Karner, and J. Francfort, "Plug-in hybrid electric vehicle charging infrastructure review," US Department of Energy-Vehicle Technologies Program, 2008.

[47] B. Gharesifard and J. Corts, "Distributed strategies for generating weight-balanced and doubly stochastic digraphs," European Journal of Control, vol. 18, no. 6, pp. 539 - 557, 2012.

[48] S. Vachirasricirikul and I. Ngamroo, "Robust controller design of heat pump and plug-in hybrid electric vehicle for frequency control in a smart microgrid based on specified-structure mixed $\mathrm{H} 2 / \mathrm{H}$ control technique," Applied Energy, vol. 88, no. 11, pp. 3860 - 3868, 2011. 


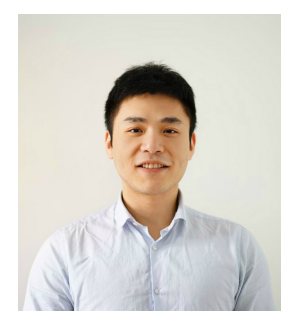

Tianqiao Zhao received his B.Eng. degree in automatic control from North China Electric Power University, Hebei, China, in 2013, and his M.Sc degree in electrical and electronic engineering from the University of Manchester, U.K., in 2014. He is now a Ph.D. candidate in control engineering with the School of Electrical and Electronic Engineering at the University of Manchester, U.K. His research interests include distributed optimization of microgrids, distributed control and energy storage systems.

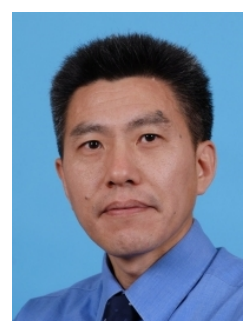

Zhengtao Ding received the B.Eng. degree from Tsinghua University, Beijing, China, and the M.Sc. degree in systems and control and the Ph.D. degree in control systems from the University of Manchester Institute of Science and Technology, Manchester, U.K. After working as a Lecturer with Ngee Ann Polytechnic, Singapore, for ten years, in 2003 , he joined The University of Manchester, Manchester, U.K., where he is currently a Senior Lecturer of control engineering with the School of Electrical and Electronic Engineering. He is the author of the book Nonlinear and Adaptive Control Systems (IET, 2013) and a number of journal papers. His research interests include nonlinear and adaptive control theory and their applications. Dr. Ding serves as an Associate Editor for the IEEE TRANSACTIONS ON AUTOMATIC CONTROL, Transactions of the Institute of Measurement and Control, Control Theory and Technology, Mathematical Problems in Engineering, Unmanned Systems, and the International Journal of Automation and Computing. 